\title{
Random walk approach to the analytic solution of random systems with multiplicative noise - the Anderson localization problem
}

\author{
V.N. Kuzovkov \\ Institute of Solid State Physics, University of Latvia, \\ 8 Kengaraga Street, LV - 1063 RIGA, Latvia \\ W. von Niessen \\ Institut für Physikalische und Theoretische Chemie, Technische Universität \\ Braunschweig, Hans-Sommer-Straße 10, 38106 Braunschweig, Germany
}

\begin{abstract}
We discuss here in detail a new analytical random walk approach to calculating the phase-diagram for spatially extended systems with multiplicative noise. We use the Anderson localization problem as an example. The transition from delocalized to localized states is treated as a generalized diffusion with a noise-induced first-order phase transition. The generalized diffusion manifests itself in the divergence of averages of wavefunctions (correlators). This divergence is controlled by the Lyapunov exponent $\gamma$, which is the inverse of the localization length, $\xi=1 / \gamma$. The appearance of the generalized diffusion arises due to the instability of a fundamental mode corresponding to correlators. The generalized diffusion can be described in terms of signal theory, which operates with the concepts of input and output signals and the filter function. Delocalized states correspond to bounded output signals, and localized states to unbounded output signals, respectively. The transition from bounded to unbounded signals is defined uniquely by the filter function $H(z)$.
\end{abstract}

Key words: Random systems, diffusion, Anderson localization, phase diagram PACS: 05.40.-a, 64.60.-i, 72.15.Rn, 71.30.+h

Email address: kuzovkov@latnet.lv (V.N. Kuzovkov). 


\section{Introduction}

A variety of phenomena in physics, chemistry and biology is modelled by stochastic differential (or difference) equations [1,2] which involve both additive and multiplicative noise. The additive noise is relatively easy to handle with the help of the central limit theorem. This type of noise disappears from the noise-averaged form of the equations. The situation changes dramatically with the appearance of multiplicative noise [3]. Some situations have been documented in the literature, in which the multiplicative noise participates in the creation of a pure noise-induced phase transition [4]. In such a case fundamental theorems of an importance comparable to the central limit theorem are still lacking. In this paper we will investigate another famous example of this phenomenon - the Anderson localization as a noise-induced metal-insulator phase transition.

Let us consider spatially extended systems with multiplicative noise. Anderson localization [5] has remained a hot topic in the physics of disordered systems for a long time (see review articles $[6,7,8]$ ) and constitutes a multidisciplinary problem. Formally the Schrödinger equation with random on-site potentials in the tight-binding representation is a stochastic algebraic equation with multiplicative noise, where physical meaning can only be attributed to certain average values (averaging over the ensemble of random potentials [9]). The problem is very similar to statistical physics, especially to the statistical physics of phase transitions (quantum phase transitions, see [10]), because there also a noise-induced metal-insulator phase transition is analyzed (keywords: critical dimension, correlation length, etc.). In the case of phase transitions one finds that the relevant parameters (in our case e.g. Lyapunov exponents or localization lengths) are not analytical functions of the disorder. Typically the theoretical analysis of disordered systems is based either on approximations or on numerical methods $[6,7]$. However, it is clear that this cannot be sufficient for systems revealing a phase transition [11], e.g. a metal-insulator transition as is the case with Anderson localization. In such a case an exact solution is greatly needed.

Disordered systems are usually considered as a part of solid state physics (or statistical physics). The theoretical methods here are very different from the ones in the theory of dynamical systems. It is known that static randomness (multiplicative noise in space) in the physics of disordered systems may drastically change macroscopic quantities. Such an effect exists also for dynamical systems with randomness in the equations of motion (multiplicative noise in time). For example, in special cases the random walk problem is mapped to a quantum mechanical tight-binding model exhibiting Anderson localization $[12,13,14]$. It might be reasonable to start from a simple microscopic stochastic kinematic model (Brownian motion and normal diffusion), and to build the 
theory of Anderson localization along this line (tight-binding model and generalized diffusion [13], with a noise-induced metal-insulator phase transition).

In our papers $[12,13]$ we presented somewhat schematically and by leaving out many mathematical details an exact analytic solution of the Anderson localization problem. Subsequently there were critical comments [15] (see also [16]) that our proof is too short and condensed, and more details are needed, the more so, since the mathematical formalism used by us is quite new for this scientific community of Anderson localization. This is why we present in the present paper the detailed derivation of the analytical solution with illustrations. Note that under the exact solution we mean the calculation of the phase diagram for the metal-insulator system $[12,13]$. We do not calculate transport and other important properties. However, the knowledge of the phase diagram permits to understand, how these properties can be calculated.

The structure of the present paper is as follows. In Section 2 we explain how the localized states can be treated in terms of a generalized diffusion. This approach allows to understand why for defining the phase diagram it is sufficient to solve exactly only equations for the joint correlators. We present and solve here the equations for arbitrary dimensions. Section 3 deals with the stability of this solution. It is shown that the generalized diffusion arises due to the divergence of the fundamental mode. The determination of the stability range of this mode permits us to calculate the phase diagram. We explain our language of input and output signals, filter function which is new for the Anderson localization community.

\section{Equations for correlators}

\subsection{General aspects}

Strictly speaking, the disordered Anderson tight-binding model [5] with Schrödinger equation

$$
\sum_{\mathcal{M}^{\prime}} \psi_{\mathcal{M}^{\prime}}=\left(E-\varepsilon_{\mathcal{M}}\right) \psi_{\mathcal{M}}
$$

where the summation over $\mathcal{M}^{\prime}$ runs over the nearest neighbours of site $\mathcal{M}=$ $\left\{m_{1}, m_{2}, \ldots, m_{D}\right\}$, cannot be solved exactly for arbitrary dimension $\mathrm{D}$. The reason is that the random potential $\varepsilon_{\mathcal{M}}$ enters the equation as a product with the random amplitude $\psi_{\mathcal{M}}$ which corresponds to the multiplicative noise case [12]. Therefore, the exact solution of eq. (1) discussed in $[12,13]$ is possible only under special circumstances to be discussed below. 
The fundamental quantity of a disordered system - the localization length $\xi$ was determined in $[12,13]$ via the Lyapunov exponent $\gamma$. In these calculations the following conditions which appear contradictory at first sight have to be satisfied. The phase diagram of the system with metal-insulator transition should be obtained in the thermodynamical limit [11] (the infinite system). However, the determination of the Lyapunov exponent needs the introduction of a coordinate system (starting point) which imposes certain limitations on the system size. Moreover, a direction for the growth of the divergent quantity should be chosen, despite the fact that all space directions are equivalent in eq. (1).

All these conditions are fulfilled for the semi-infinite system, or system with a boundary, where the index $n \equiv m_{D} \geq 0$, but all $m_{j} \in(-\infty, \infty), j=$ $1,2, \ldots, p$, with $p=D-1$. The boundary which is the layer $n=0$ defines the preferential direction (the axis $n$ ) along which the Lyapunov exponent $\gamma$ will be calculated.

It is convenient to interpret the index $n=0,1, \ldots, \infty$ as the discrete-time, whereas all other indices combine in the vector $\mathbf{m}=\left\{m_{1}, m_{2}, \ldots, m_{p}\right\}$. The Schrödinger equation (1) can be rewritten as a recursion equation (in terms of the discrete-time, and assuming summation over repeated indices)

$$
\psi_{n+1, \mathbf{m}}=-\varepsilon_{n, \mathbf{m}} \psi_{n, \mathbf{m}}-\psi_{n-1, \mathbf{m}}+\mathcal{L}_{\mathbf{m}, \mathbf{m}^{\prime}} \psi_{n, \mathbf{m}^{\prime}}
$$

where the operator

$$
\mathcal{L}_{\mathbf{m}, \mathbf{m}^{\prime}}=E \delta_{\mathbf{m}, \mathbf{m}^{\prime}}-\sum_{\mathbf{m}^{\prime \prime}} \delta_{\mathbf{m}^{\prime \prime}, \mathbf{m}^{\prime}}
$$

is introduced for compactness (summation over $\mathbf{m}^{\prime \prime}$ includes the nearest neighbours of the site $\mathbf{m}$ ). The discrete-time equation (2) is a difference equation of the second order, which needs two initial conditions (Cauchy problem for the difference equation). The first natural condition is $\psi_{0, \mathbf{m}}=0$. The second initial condition can be presented in the general form $\psi_{1, \mathbf{m}}=\alpha_{\mathbf{m}}$ without any additional conditions for the arbitrary constants $\alpha_{\mathbf{m}}$, except that they are supposed to be finite. The rules for the treatment of the field $\alpha_{\mathbf{m}}$ will be explained below, section 3.3.

The recursion equation (2) reveals a general feature of the causality - in its formal solution $\psi_{n+1, \mathbf{m}}$ depends only on the random variables $\varepsilon_{n^{\prime}, \mathbf{m}^{\prime}}$ with $n^{\prime} \leq$ $n$. This gives us a hint for the exact solution of the equation provided that random variables $\varepsilon_{\mathcal{M}}$ on different sites do not correlate: $\left\langle\varepsilon_{\mathcal{M}_{\mathcal{M}}} \varepsilon_{\mathcal{M}^{\prime}}\right\rangle \propto \delta_{\mathcal{M}, \mathcal{M}^{\prime}}$. In this case all amplitudes $\psi$ on the r.h.s. of eq. (2) are statistically independent of $\varepsilon_{n, \mathbf{m}}$. In other words, while performing the mathematical operations in eq. (2), e.g. taking the square of both equation sides, with a further averaging 
over an ensemble of different realizations of the random potentials (symbol $\langle\ldots\rangle)$, the average of the product of amplitudes $\psi$ and potentials $\varepsilon_{n, \mathbf{m}}$ can be replaced by a product of the corresponding average quantities. Therefore, the exact solution can be obtained only when the on-site potentials $\varepsilon_{n, m}$ are independently and identically distributed. We assume hereafter existence of

the two first moments, $\left\langle\varepsilon_{n, \mathbf{m}}\right\rangle=0$ and $\left\langle\varepsilon_{n, \mathbf{m}}^{2}\right\rangle=\sigma^{2}$, where the parameter $\sigma$ characterizes the disorder level.

\subsection{Generalized diffusion}

The ensemble averaging using recursion eq.(2) allows us to calculate averages of different functions containing the amplitudes $\psi$. The problem arises, which averages we can and should calculate? E.g. several even moments of the amplitude were calculated in the 1-D case [17], whereas only the second momentum was calculated for multidimensional systems in $[12,13]$. It is generally believed that the complete information is available from the complete set of all moments [18]. However, it is not clear, how this information should be analyzed. In particular, how can the one parameter of our interest - the localization length $\xi$ - be extracted from the infinite set of amplitude momenta?

It was suggested in [15] to calculate not only the second, $\left\langle|\psi|^{2}\right\rangle$, and other momenta, but also physical values, such as $\langle\ln |\psi|\rangle$. The average quantities are divided in [15] into two categories: containing physical information (e.g. $\langle\ln |\psi|\rangle)$ and containing no physical information (e.g. $\left.\left\langle|\psi|^{2}\right\rangle\right)$.

In other words, one has to understand how the choice of a semi-infinite system with a selected direction ( $n$ axis) and the boundary (layers in the transversal directions with $n=0,1$ ) allows us to detect the localized states under question.

Let us consider for simplicity a 1-D system, where the amplitude $\psi=\psi_{n}$ and the second initial condition $\psi_{1}=\alpha$. Again, for simplicity let us consider $\alpha$ to be a real quantity, i.e. $|\psi|^{2}=\psi^{2}$. The recursive equation reads:

$$
\psi_{n+1}=\left(E-\varepsilon_{n}\right) \psi_{n}-\psi_{n-1},
$$

where $\left\langle\varepsilon_{n}\right\rangle=0,\left\langle\varepsilon_{n}^{2}\right\rangle=\sigma^{2}$.

Let us consider now the random walk problem (Brownian motion $[1,19]$ for the coordinate $\psi_{n}$ ) which is described by the following equations

$$
\psi_{n+1}=\psi_{n}+\varepsilon_{n}
$$

There exists a mean squared displacement $\left\langle\varepsilon_{n}^{2}\right\rangle=\sigma^{2}$ of the Brownian particle 
during a step.

Considering $n$ as the discrete-time index, both eqs. (4) and (5) describe the dynamics of the system with a stochastic time-dependent perturbation $\varepsilon_{n}$. The only difference is that the dynamics of the system (5) is trivial: $\psi_{n} \equiv \psi_{0}$ when there is no perturbation, $\varepsilon_{n}=0$. In contrast, the dynamical system (4) even for $\varepsilon_{n}=0$ reveals proper dynamics (it is a second order equation!). In the band region, $|E|<2$, this corresponds to the bounded motion. Therefore, the proper dynamics of both systems corresponds to the bounded trajectories, $\psi_{n}^{2}<\infty$.

Assuming now that $\sigma \neq 0$, eq. (5) describes the normal diffusion. The diffusion motion is characterized by divergences, e.g. for the mean time when the system returns to the initial state. In particular, the momenta of the amplitude $\psi_{n}$ are also divergent with the discrete-time $n$, e.g.

$$
\left\langle\psi_{n}^{2}\right\rangle=\psi_{0}^{2}+\sigma^{2} n
$$

reveals a power law divergence. For normal diffusion the mean square displacement $\left\langle\psi_{n}^{2}\right\rangle$ of the Brownian particles is linear in time.

To detect the diffusion, it is sufficient to demonstrate the divergence of the second moment of the amplitude and to establish its law of time-dependence [20,21]. For example, for anomalous diffusion the mean quare displacement $\left\langle\psi_{n}^{2}\right\rangle$ of the particles is no longer linear in time, but follows a generalized Fick's second law [22].

Generally speaking, other moments contain additional information, which, however, is not important. The divergence of the second moment defines the conditions of the diffusion appearance: if the second moment is divergent, so are all other even moments (this is why the choice of a particular moment for further analysis is not unique). The law of the divergence of the timedependence allows us to distinguish between normal and abnormal diffusion. Important is the fact that the second moment $\left\langle\psi_{n}^{2}\right\rangle$ in eq. (5) can be found exactly analytically and thus we prefer its use $\left(\psi^{2}\right.$-definition). Speaking formally, the diffusion could also be classified using other averaged quantities, e.g. $\left\langle\left|\psi_{n}\right|\right\rangle$, but such a choice is not convenient for mathematical reasons; it hinders an analytical solution.

As is well-known, in a 1-D system described by eq. (4) any disorder $\sigma \neq 0$ leads to localization. This manifests itself in the simultaneous divergence, as $n \rightarrow \infty$, of different average quantities of $\left|\psi_{n}\right|$, e.g. the linear divergence of $\left\langle\ln \left|\psi_{n}\right|\right\rangle$ (log-definition of localization), whereas the exponential divergence occurs for the powers of $\left|\psi_{n}\right|[17]$. The appearance of the localization in the approach based on eq. (4) is equivalent to the appearance of diffusion. In eq. (5) the 

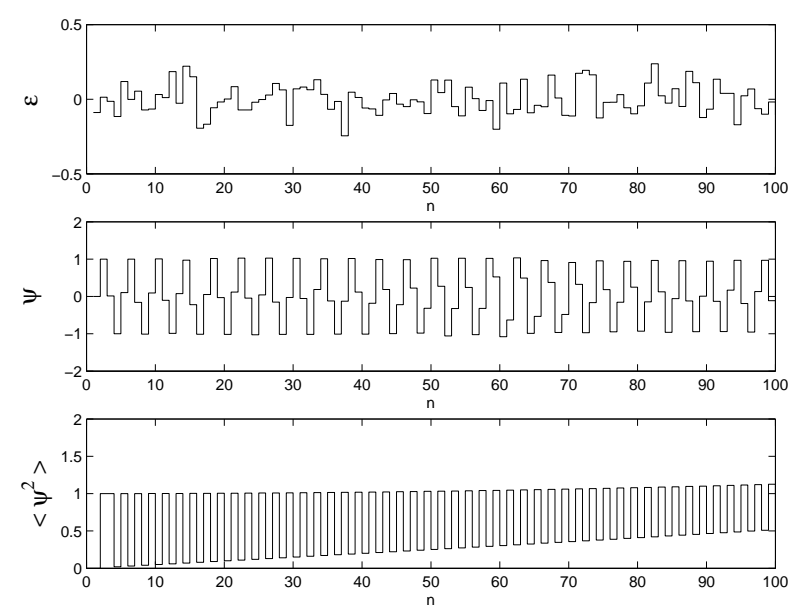

Fig. 1. Exponential divergence of second moment for generalized diffusion, eq. (4), for $\sigma=0.1$ and $E=0$.

random perturbation $\varepsilon_{n}$ is additive, which determines the linear (power-law) character of the second moment divergence (normal diffusion). In contrast, eq. (4) contains the product of $\varepsilon_{n}$ and $\psi_{n}$ (multiplicative noise) which determines the exponential character of the divergence (see also Brownian motion [3] and normal nonlinear diffusion [23] with multiplicative noise).

I.e., we can speak of the generalized diffusion, analyzing the $\left\langle\psi_{n}^{2}\right\rangle=f(n)$ divergence as a function of $n$. The exponential localization here corresponds to the exponential growth of $f(n) \propto \exp (2 \gamma n)$, Fig. 1 , where $\xi=1 / \gamma$ can be interpreted as the localization length $\left(\psi^{2}\right.$ - localization definition $\left.[12,13,17]\right)$. On the one hand, the numerical solution for the amplitude $\psi_{n}$ are plotted here using eq.(4) together with arbitrary realizations of perturbations $\varepsilon_{n}$ (two upper windows). On the other hand, the lower window of Fig.1 show the exact analytical solution for the second momenta $\left\langle\psi_{n}^{2}\right\rangle$ (eqs.(51) and (53) of this paper). The analysis of these results (as well as other data for different $E$ and $\sigma)$ demonstrates that purely exponential behaviour, $\left\langle\psi_{n}^{2}\right\rangle \propto \exp (2 \gamma n)$, is valid only asymptotically, as $n \rightarrow \infty$. In other words, the exponential behaviour of divergence is understood by its definition, eq.(49). There is a transient regime for finite $n$ values especially for small $\gamma$ (as shown in Fig.1) where the exponential behaviour is combined with the oscillations corresponding to the proper dynamics, eq.(5). Note that the bounded proper motion of $\psi_{n}$ for $\varepsilon_{n}=0$ in the band region, $|E|<2$, corresponds to oscillating functions.

The $\psi^{2}$-definition is convenient not only because the equations for the second moment can be exactly solved (see below). It is important that this definition works also for the non-exponential localization, which corresponds to the non-exponential behaviour of $f(n)$. In contrast, the traditional log-definition is valid only for the exponential localization, and does not allow an exact analytical solution. 
It was analytically shown [17] for 1-D systems that if the higher moments of the random potential $\varepsilon_{n}$ can be neglected (for $\sigma \rightarrow 0$ ) and we can restrict ourselves to the only parameter $\sigma$, the exponents in all even moments (as well as in log-definition) are proportional to each other, they differ only by numerical factors. The proportionality of the different exponents (Lyapunov exponents) in the exponential growth indicates that different definitions of the generalized diffusion are in fact identical and one can use any of them, e.g the $\psi^{2}$-definition.

Note that the emergence of the generalized diffusion phenomenon and the existence of the phase diagrams in dynamical systems with the proper dynamics are analogous to critical phenomena in equilibrium and non-equilibrium systems. In this sense, it is clear that use of the $\psi^{2}$-definition for the determination of the phase-diagram is quite sufficient. Exactly solvable problems in the physics of phase transitions [11], e.g. the Ising model, have demonstrated that in spite of the fact that the physical quantities, i.e. long range order parameter, magnetic susceptibility, specific heat, etc. are defined as different averages, they still behave similarly, when only the phase-diagram is considered. This is not surprising since one deals here with averages obtained for the same statistical ensemble. In particular, the critical temperature obtained for the order parameter coincides with that obtained for the susceptibility. This occurs for the exact solutions, although deviations can arise for the approximate solutions. Similarly, the phase diagram for the system with a metal-insulator transition can be defined uniquely if one determines any non-trivial average quantity. This is why the choice of $\langle\ln |\psi|\rangle$ is not convenient. A product of a sum of variables can be represented as a polynomial, where each term can be calculated, whereas the logarithm of a sum cannot be represented this way and thus the causality principle cannot be used. On the other hand, the averages of the powers of the amplitude can be calculated exactly.

When we speak of the phase-diagram, we mean determination of the boundaries of metallic and insulating phases. In this respect, the results $[12,13]$ demonstrate that the second order moments form a closed and linear set of equations and, where the only characteristic of the random potentials is the second momentum, $\sigma^{2}$, have a simple interpretation. The phase boundaries are defined by the only simple parameter $\sigma$ of the random potential. That is, calculation of second moments (correlators) is the direct way to obtaining the phase diagram.

\subsection{Correlators}

In general, the field $\alpha_{\mathbf{m}}$ is complex. We can introduce the simplest non-trivial correlators based on the $\psi^{2}$-definition: 


$$
\begin{array}{r}
x(n)_{\mathbf{m}, \mathbf{l}}=\left\langle\psi_{n, \mathbf{m}}^{*} \psi_{n, \mathbf{l}}\right\rangle, \\
y(n)_{\mathbf{m}, \mathbf{l}}=\frac{1}{2}\left[\left\langle\psi_{n, \mathbf{m}}^{*} \psi_{n-1, \mathbf{l}}\right\rangle+\left\langle\psi_{n-1, \mathbf{m}}^{*} \psi_{n, \mathbf{l}}\right\rangle\right] .
\end{array}
$$

Let us for mathematical simplicity consider real $\alpha_{\mathbf{m}}$. This is also justified by the fact that the final eqs. (36),(37) remain the same. For real $\alpha_{\mathbf{m}}$ definitions can be simplified:

$$
\begin{array}{r}
x(n)_{\mathbf{m}, \mathbf{l}}=\left\langle\psi_{n, \mathbf{m}} \psi_{n, \mathbf{l}}\right\rangle, \\
y(n)_{\mathbf{m}, \mathbf{l}}=\left\langle\psi_{n, \mathbf{m}} \psi_{n-1, \mathbf{l}}\right\rangle .
\end{array}
$$

Taking square of the both sides of the eq. (2) and using the causality principle, one gets

$$
\begin{array}{r}
x(n+1)_{\mathbf{m}, \mathbf{l}}=\delta_{\mathbf{m}, \mathbf{l}} \sigma^{2} x(n)_{\mathbf{m}, \mathbf{m}}+x(n-1)_{\mathbf{m}, \mathbf{l}}+ \\
\mathcal{L}_{\mathbf{m}, \mathbf{m}^{\prime}} x(n)_{\mathbf{m}^{\prime}, \mathbf{l}^{\prime}} \mathcal{L}_{\mathbf{l}^{\prime}, \mathbf{l}}-\mathcal{L}_{\mathbf{m}, \mathbf{m}^{\prime}} y(n)_{\mathbf{m}^{\prime}, \mathbf{l}}-\mathcal{L}_{\mathbf{l}, \mathbf{l}^{\prime}} y(n)_{\mathbf{l}^{\prime}, \mathbf{m}}
\end{array}
$$

Analogously one obtains the additional relation

$$
y(n+1)_{\mathbf{m}, \mathbf{l}}=-y(n)_{\mathbf{l}, \mathbf{m}}+\mathcal{L}_{\mathbf{m}, \mathbf{m}^{\prime}} x(n)_{\mathbf{m}^{\prime}, \mathbf{l}} .
$$

Note that eq.(11) contains explicitly the diagonal correlators with $\mathbf{l}=\mathbf{m}$ : $\chi(n)_{\mathbf{m}}=x(n)_{\mathbf{m}, \mathbf{m}}$. It is essential that diagonal correlators are positive numbers, $\chi(n)_{\mathbf{m}} \geq 0$.

\subsection{Z-transform}

It is convenient to use along the $n$ axis the Z-transform which is common in discrete-time systems [24]:

$$
\begin{aligned}
& X(z)_{\mathbf{m}, \mathbf{l}}=\sum_{n=0}^{\infty} \frac{x(n)_{\mathbf{m}, \mathbf{l}}}{z^{n}} \\
& Y(z)_{\mathbf{m}, \mathbf{l}}=\sum_{n=0}^{\infty} \frac{y(n)_{\mathbf{m}, \mathbf{l}}}{z^{n}} .
\end{aligned}
$$

After transformation one gets

$$
\begin{array}{r}
\left(z-z^{-1}\right) X(z)_{\mathbf{m}, \mathbf{l}}-\sigma^{2} \delta_{\mathbf{m}, \mathbf{l}} \chi(z)_{\mathbf{m}}-x(1)_{\mathbf{m}, \mathbf{l}}= \\
\mathcal{L}_{\mathbf{m}, \mathbf{m}^{\prime}} X(z)_{\mathbf{m}^{\prime}, \mathbf{l}^{\prime}} \mathcal{L}_{\mathbf{l}^{\prime}, \mathbf{l}}-\mathcal{L}_{\mathbf{m}, \mathbf{m}^{\prime}} Y(z)_{\mathbf{m}^{\prime}, \mathbf{l}}-\mathcal{L}_{\mathbf{l}, \mathbf{l}^{\prime}} Y(z)_{\mathbf{l}^{\prime}, \mathbf{m}}, \\
z Y(z)_{\mathbf{m}, \mathbf{l}}=-Y(z)_{\mathbf{l}, \mathbf{m}}+\mathcal{L}_{\mathbf{m}, \mathbf{m}^{\prime}} X(z)_{\mathbf{m}^{\prime}, \mathbf{l}}
\end{array}
$$


Hereafter the symmetry properties of the operator $\mathcal{L}_{\mathbf{m}, \mathbf{l}}$ are used.

The solution of the second equation is simple:

$$
Y(z)_{\mathbf{m}, \mathbf{l}}=\frac{z}{z^{2}-1} \mathcal{L}_{\mathbf{m}, \mathbf{m}^{\prime}} X(z)_{\mathbf{m}^{\prime}, \mathbf{l}}-\frac{1}{z^{2}-1} X(z)_{\mathbf{m}, \mathbf{l}^{\prime}} \mathcal{L}_{\mathbf{l}^{\prime}, \mathbf{l}}
$$

which leads to the equation for $X$-correlators

$$
\begin{aligned}
\left(z-z^{-1}\right) X(z)_{\mathbf{m}, \mathbf{l}}- & \sigma^{2} \delta_{\mathbf{m}, \mathbf{l}} \chi(z)_{\mathbf{m}}-x(1)_{\mathbf{m}, \mathbf{l}}=\frac{z^{2}+1}{z^{2}-1} \mathcal{L}_{\mathbf{m}, \mathbf{m}^{\prime}} X(z)_{\mathbf{m}^{\prime}, \mathbf{l}^{\prime}} \mathcal{L}_{\mathbf{l}^{\prime}, \mathbf{l}}(18) \\
& -\frac{z}{z^{2}-1}\left[\mathcal{L}_{\mathbf{m}, \mathbf{m}^{\prime}} \mathcal{L}_{\mathbf{m}^{\prime}, \mathbf{l}^{\prime}} X(z)_{\mathbf{l}^{\prime}, \mathbf{l}}+X(z)_{\mathbf{m}, \mathbf{m}^{\prime}} \mathcal{L}_{\mathbf{m}^{\prime}, \mathbf{l}^{\prime}} \mathcal{L}_{\mathbf{l}^{\prime}, \mathbf{l}}\right]
\end{aligned}
$$

Taking into account the second initial condition, $x(1)_{\mathbf{m}, \mathbf{l}}=\alpha_{\mathbf{m}} \alpha_{\mathbf{l}}$.

\subsection{Fourier transform}

The obtained equation can be easily solved formally via Fourier expansion. Indeed, one gets for diagonal correlators

$$
\begin{array}{r}
\chi(z)_{\mathbf{m}}=\int \frac{d^{p} \mathbf{k}}{(2 \pi)^{p}} \chi(z, \mathbf{k}) e^{-i \mathbf{k m}}, \\
\chi(z, \mathbf{k})=\sum_{\mathbf{m}} \chi(z)_{\mathbf{m}} e^{i \mathbf{k m}} .
\end{array}
$$

A general equation for $X$-correlators has to be solved using double Fourier expansion:

$$
X\left(z, \mathbf{k}, \mathbf{k}^{\prime}\right)=\sum_{\mathbf{m}, \mathbf{l}} X(z)_{\mathbf{m}, \mathbf{l}} e^{i \mathbf{k} \mathbf{m}+i \mathbf{k}^{\prime} \mathbf{l}}
$$

This leads to

$$
U\left(z, \mathbf{k}, \mathbf{k}^{\prime}\right) X\left(z, \mathbf{k}, \mathbf{k}^{\prime}\right)=\alpha(\mathbf{k}) \alpha\left(\mathbf{k}^{\prime}\right)+\sigma^{2} \chi\left(z, \mathbf{k}+\mathbf{k}^{\prime}\right)
$$

where

$$
U\left(z, \mathbf{k}, \mathbf{k}^{\prime}\right)=\left(z-z^{-1}\right)-\frac{z^{2}+1}{z^{2}-1} \mathcal{L}(\mathbf{k}) \mathcal{L}\left(\mathbf{k}^{\prime}\right)+\frac{z}{z^{2}-1}\left[\mathcal{L}^{2}(\mathbf{k})+\mathcal{L}^{2}\left(\mathbf{k}^{\prime}\right)\right]
$$


and

$$
\alpha(\mathbf{k})=\sum_{\mathbf{m}} \alpha_{\mathbf{m}} e^{i \mathbf{k m}}
$$

The function

$$
\mathcal{L}(\mathbf{k})=E-2 \sum_{j=1}^{p} \cos \left(k_{j}\right)
$$

arises as the Fourier transform of the operator $\mathcal{L}_{\mathbf{m}, 1}$.

\subsection{Diagonal correlators}

Assuming $\mathbf{k}^{\prime \prime}=\mathbf{k}+\mathbf{k}^{\prime}$, eq.(22) can be rewritten as

$$
U\left(z, \mathbf{k}, \mathbf{k}^{\prime \prime}-\mathbf{k}\right) X\left(z, \mathbf{k}, \mathbf{k}^{\prime \prime}-\mathbf{k}\right)=\alpha(\mathbf{k}) \alpha\left(\mathbf{k}^{\prime \prime}-\mathbf{k}\right)+\sigma^{2} \chi\left(z, \mathbf{k}^{\prime \prime}\right) .
$$

Using the relation

$$
\chi\left(z, \mathbf{k}^{\prime \prime}\right)=\int \frac{d^{p} \mathbf{k}}{(2 \pi)^{p}} X\left(z, \mathbf{k}, \mathbf{k}^{\prime \prime}-\mathbf{k}\right),
$$

eq.(26) can be solved elementary for diagonal correlators:

$$
\begin{array}{r}
\chi(z, \mathbf{k})=\mathcal{H}(z, \mathbf{k}) \chi^{(0)}(z, \mathbf{k}), \\
\chi^{(0)}(z, \mathbf{k})=\int \frac{d^{p} \mathbf{k}^{\prime}}{(2 \pi)^{p}} \frac{\alpha\left(\mathbf{k}^{\prime}\right) \alpha\left(\mathbf{k}-\mathbf{k}^{\prime}\right)}{U\left(z, \mathbf{k}^{\prime}, \mathbf{k}-\mathbf{k}^{\prime}\right)} \\
\frac{1}{\mathcal{H}(z, \mathbf{k})}=1-\sigma^{2} \int \frac{d^{p} \mathbf{k}^{\prime}}{(2 \pi)^{p}} \frac{1}{U\left(z, \mathbf{k}^{\prime}, \mathbf{k}-\mathbf{k}^{\prime}\right)}
\end{array}
$$

Therefore we have obtained for an arbitrary second initial condition (field $\alpha_{\mathbf{m}}$ ) an exact solution for fundamental averages - diagonal correlators. After use of the Fourier transform the solution is expanded in modes labelled by the $\mathbf{k}$-vector and describing the oscillations in the solutions in the transversal direction. It is easy to see that the $\psi^{2}$-definition leads to equations containing only the $\sigma$ parameter of the random potentials. If $\sigma=0$, all functions $\mathcal{H}(z, \mathbf{k}) \equiv 1$ and thus $\chi(z, \mathbf{k}) \equiv \chi^{(0)}(z, \mathbf{k})$. In other words, functions $\chi^{(0)}(z, \mathbf{k})$ correspond to solutions in a completely ordered system. Note that the boundary conditions (field $\alpha_{\mathbf{m}}$ ) influence only functions $\chi^{(0)}(z, \mathbf{k})$ which are independent of the parameter $\sigma$. Introduction of disorder, $\sigma>0$, transforms the 
initial solution $\chi^{(0)}(z, \mathbf{k})$ into $\chi(z, \mathbf{k})$, where the operator $\mathcal{H}(z, \mathbf{k})$ describes this transformation.

In our derivation we assumed the system size in a transversal direction to be infinite, $m_{j} \in(-\infty, \infty)$, and thus the equations obtained correspond to the thermodynamic limit $(L=\infty)$. In the case of finite size of the system, $m_{j}=0,1, \ldots, L-1$ (cyclic conditions) the integrals should be replaced by series. Let us consider for illustration the 2 -D case: $p=D-1 \equiv 1$. Here the index $\mathbf{m}=m$ has $L$ values and the number of modes $\mathbf{k}=k$ also equals $L$. That is, analysis of the diagonal correlators leads to $L$-order matrices (vectors). Surprisingly, this obvious fact was interpreted in ref. [15] that degrees of freedom of the initial problem were lost in our paper [12], thus questioning our proof of the Anderson theorem. This conclusion was based on the traditional approach of the transfer-matrix $[15,18]$ which operates with much larger, $L^{2} \times L^{2}$, matrices for 2-D. The transition from $L^{2} \times L^{2}$ matrices to $L$ order matrices was interpreted as an error arising due to averaging over initial conditions [12] and thus artificially introducing a translation in the transversal directions. However, we do not use in the present study such an averaging (see also Section 3.6). The $L$-dimensional matrices are the natural mathematical tool for the description of diagonal correlators. Moreover, an excessive size of $L^{2} \times L^{2}$ matrices indicates that the transfer-matrix formalism is not adequate. This is seen, in particular, from the existence of the so-called trivial eigenvalues of the transfer-matrix $[15,18]$, which are $\sigma$-independent. Moreover, the transfer-matrix does not permit to make the transition to the thermodynamic limit and thus to calculate the phase-diagram (for more details see [16]). These disadvantages are absent in the 1 -D case, when formally $L=0$ and the matrix dimensions coincide. This is why we used in [12] the transfer-matrix method only in the 1-D case and exclusively for the illustration.

\section{Anderson localization and stability}

\subsection{Signals and filters}

The set of equations for the correlators is linear, the same is true for eqs.(28) for each $\mathbf{k}$-mode. The modes are normal (no mixing of modes with different $\mathbf{k}$ values). The above mentioned divergence (exponential or non-exponential growth) of the diagonal correlators, arising due to localized states, transforms mathematically to the instability of the set of linear equations. For linear discrete-time systems under study the most adequate formalism is signal theory [24]. Following $[12,13,24]$, let us define $\chi^{(0)}(z, \mathbf{k})$ as input signals. In our particular case this is a mathematical characteristic of the $\mathbf{k}$-mode for an initial ordered systems. (Its sense is explained below). The inverse Z-transform 
of a given mode can be performed as follows:

$$
\chi^{(0)}(z, \mathbf{k}) \Rightarrow \chi^{(0)}(n, \mathbf{k}) .
$$

The input signal is a one dimensional numerical series. The $\chi(z, \mathbf{k})$ is the output signal; respectively after the inverse Z-transform the latter corresponds to the numerical series $\chi(n, \mathbf{k})$, associated with the disordered system, $\sigma>0$. The relation between these two signals is given by eq.(28): the output signals are linear transformations of input signals performed through $\mathcal{H}(z, \mathbf{k})$ functions called the system filter [24].

Physical systems with time as independent variable are causal systems [24]. In our problem with discrete-time index $n$ the causality is of primary importance for the interpretation of the result. Note that in the general case the inverse Z-transform is defined through the complex integral

$$
h(n, \mathbf{k})=\frac{1}{2 \pi i} \oint \mathcal{H}(z, \mathbf{k}) z^{n} \frac{d z}{z} .
$$

The integration here is performed over the complex plane called the region of convergence (ROC) [12,24], well-defined for the causal filters. For the causal systems $h(n, \mathbf{k})=0$ for $n<0$ holds, thus after the inverse Z-transform eqs.(28) transform into the convolution property [24]:

$$
\chi(n, \mathbf{k})=\sum_{l=0}^{n} h(n-l, \mathbf{k}) \chi^{(0)}(l, \mathbf{k}) .
$$

One can see here the linear transformation of input signals into output signals. The fundamental property of the signal theory is that the divergence of the output signals is related to the asymptotic behaviour of the filter coefficients $h(n, \mathbf{k})$ as $n \rightarrow \infty$, which is mathematically equivalent to the poles of the $\mathcal{H}(z, \mathbf{k})$ function of the complex argument $z[24]$.

\subsection{Fundamental mode}

Since the appearance of localized states leads to the divergence of the diagonal correlator, it is necessary to clarify, which fundamental mode $\mathbf{k}=\mathbf{k}_{\mathbf{0}}$ looses its stability and thus is responsible for the divergence. Such a problem is quite general in many branches of physics. However, in our case it is quite obvious that the fundamental mode is static, $\mathbf{k}_{\mathbf{0}}=\mathbf{0}$.

The mode with $\mathbf{k} \neq \mathbf{0}$ describes transversal oscillations of the diagonal correlators, but the sign of the divergent oscillating solution is not defined (see 
also section 3.6). On the other hand, signs of the diagonal correlators are well defined, they are non-negative, $\chi(n)_{\mathbf{m}} \geq 0$. That is, in our particular case the solution is trivial, the fundamental mode is static. It is thus necessary to study the boundaries of the stability of the fundamental mode $\mathbf{k}_{\mathbf{0}}=\mathbf{0}$ only (the sign of the divergent non-oscillating solution is defined), which means uniquely the determination of the phase diagram of the system.

Assuming $\left.\chi^{(0)}(z, \mathbf{0}) \equiv S^{(0)}(z), \chi(z, \mathbf{0}) \equiv S(z), \mathcal{H}(z, \mathbf{0}) \equiv H(z)\right)$, we arrive at

$$
S(z)=H(z) S^{(0)}(z) .
$$

After the inverse Z-transform one gets

$$
s_{n}=\sum_{l=0}^{n} h_{n-l} s_{l}^{(0)}
$$

The fundamental input signal $S^{(0)}(z)$ here and the fundamental filter are defined by the integrals:

$$
\begin{array}{r}
S^{(0)}(z)=\frac{(z+1)}{(z-1)} \int \frac{d^{p} \mathbf{k}}{(2 \pi)^{p}} \frac{|\alpha(\mathbf{k})|^{2}}{\left[(z+1)^{2} / z-\mathcal{L}^{2}(\mathbf{k})\right]} \\
\frac{1}{H(z)}=1-\sigma^{2} \frac{(z+1)}{(z-1)} \int \frac{d^{p} \mathbf{k}}{(2 \pi)^{p}} \frac{1}{\left[(z+1)^{2} / z-\mathcal{L}^{2}(\mathbf{k})\right]}
\end{array}
$$

Note also the relation

$$
h_{n}=\frac{1}{2 \pi i} \oint H(z) z^{n} \frac{d z}{z} .
$$

That is, we have got here a new derivation of relations earlier derived in Refs. $[12,13]$. Note that for the $1-\mathrm{D}$ case the integral disappears, since $p=0$, and one gets

$$
\begin{gathered}
S^{(0)}(z)=\frac{(z+1)}{(z-1)} \frac{|\alpha|^{2}}{\left[(z+1)^{2} / z-E^{2}\right]}, \\
\frac{1}{H(z)}=1-\sigma^{2} \frac{(z+1)}{(z-1)} \frac{1}{\left.\left[(z+1)^{2} / z-E^{2}\right)\right]}
\end{gathered}
$$

\subsection{Fundamental input signal}

Let us discuss the physical sense of the input signal signal $S^{(0)}(z)$ (or $\left.s_{n}^{(0)}\right)$. Note that this corresponds to an ideal system with $\sigma=0$. When there is 
no disorder, $\varepsilon_{\mathcal{M}} \equiv 0$, the particular solutions of the tight-binding eq. (1) are bounded functions, plane waves $\exp (i \mathcal{K} \mathcal{M})$ with $\mathcal{K}=\left\{k_{1}, k_{2}, \ldots, k_{D}\right\}$, provided for a given energy $E$

$$
\mathcal{E}(\mathcal{K})=E
$$

holds, where

$$
\mathcal{E}(\mathcal{K})=\sum_{j=1}^{D} 2 \cos \left(k_{j}\right),
$$

otherwise particular solutions are not bounded functions, which lie beyond the band and have no physical interpretation. If we divide the wave vector into transversal or normal directions, $\mathcal{K} \equiv\left\{\mathbf{k}, k_{D}\right\}$, and keeping in mind that

$$
\mathcal{L}(\mathbf{k})=2 \cos \left(k_{D}\right)
$$

for a given transversal mode $\mathbf{k}$ the bounded physical solution exists, provided $|\mathcal{L}(\mathbf{k})| \leq 2$. These relations are sufficient for understanding the physical sense of the input signal.

Eq. (36) contains in the integrand the function

$$
\frac{(z+1)}{(z-1)} \frac{1}{(z+1)^{2} / z-\mathcal{L}^{2}(\mathbf{k})} .
$$

Under the condition $|\mathcal{L}(\mathbf{k})| \leq 2$ its inverse Z-transform gives

$$
\frac{\sin ^{2}\left(k_{D} n\right)}{\sin ^{2}\left(k_{D}\right)}
$$

where $k_{D}=k_{D}(\mathbf{k})$ is the solution of eq.(43). Assuming that the arbitrary field $\alpha_{\mathbf{m}}$ is selected in such a way that the Fourier transform coefficients $\alpha(\mathbf{k})$ are not zero only if $|\mathcal{L}(\mathbf{k})| \leq 2$, eq.(36) after the Z-transform reads

$$
s_{n}^{(0)}=\int \frac{d^{p} \mathbf{k}}{(2 \pi)^{p}} \frac{|\alpha(\mathbf{k})|^{2} \sin ^{2}\left(k_{D} n\right)}{\sin ^{2}\left(k_{D}\right)} .
$$

The $s_{n}^{(0)}$ is bounded for any $n$.

On the other hand, assuming that $|\mathcal{L}(\mathbf{k})| \leq 2$ is violated for certain $\mathbf{k}$, so the $\alpha(\mathbf{k}) \neq 0$ for $|\mathcal{L}(\mathbf{k})| \geq 2$, the inverse Z-transform of eq.(44) leads to the function 


$$
\frac{\sinh ^{2}\left(\kappa_{D} n\right)}{\sinh ^{2}\left(\kappa_{D}\right)}
$$

which increases without bounds as $n \rightarrow \infty$. Here $\kappa_{D}=\kappa_{D}(\mathbf{k})$ is the solution of equation

$$
|\mathcal{L}(\mathbf{k})|=2 \cosh \left(\kappa_{D}\right)
$$

The input signal $s_{n}^{(0)}$ is also increasing to infinity as a function of $n$. Note that both input and output signals are real values.

In other words, on can conclude that any physical solution of an ideal system $(\sigma=0)$ with bounded wave functions has a one-to-one correspondence to the mathematical object - a bounded 1-D input signal $s_{n}^{(0)}$. Such solutions always exist, if the energy $E$ lies within the band interval. On the other hand, formal solutions without physical interpretation (unbounded solutions beyond the band) correspond to the 1-D unbounded input signals. Note that particular numerical values of input or output signals are not important from the point of view of the signal theory [24] whose main concern is a qualitative discrimination between bounded and unbounded signals.

\subsection{Fundamental filter}

The quantity characterizing the phase diagram of a disordered system is the fundamental filter, eq.(37). Its idea is quite clear [12,13]. Let us consider the physical states inside the band, $|E|<2 D$. All these states are described by wave functions bounded in amplitude, which corresponds to a bounded 1-D input signal $s_{n}^{(0)}$. In a disordered system this signal transforms into $s_{n}$, according to eq. (35). This output signal $s_{n}$ can be either bounded, or infinitely growing (generalized diffusion).

The natural interpretation of bounded output signals is that they - as before - correspond to the delocalized states bounded in amplitude. The unbounded output signals correspond to the localized states, respectively, which lead in the semi-infinite system to the divergence of the diagonal correlators. An important result of signal theory [24] is that the divergence does not result from properties of a particular bounded input signal; the cause lies in the filter, $H(z)$ or $h_{n}$. We define the phase diagram of the Anderson model based on a general concept of the signal theory known as a BIBO stability [24]. Namely, a system is BIBO stable if every Bounded Input leads to a Bounded Output. A stable system (delocalized states) is characterized by a stable filter $H(z)$; its main property is the absence of poles in the complex $z$-plane outside the circle $|z|>1$. An unstable system (localized states) is characterized by the 
unstable filter $H(z)$ with poles outside the circle $|z|>1$. As was shown in $[12,13]$, in the Anderson model the pole $z_{0}$ of the unstable filter lies on the real axis. Consequently, $z_{0}=\exp (2 \gamma)$, where $\gamma$ is the Lyapunov exponent $\left(\psi^{2}\right.$-definition) $[12,13]$. Using the inverse Z-transform, one can easily find that asymptotically $h_{n} \sim \exp (2 \gamma n)$, i.e. it is divergent. The localization length is defined as $\xi=\gamma^{-1}$.

The mathematical formalism of the signal theory allows to extend and complement this result for the energy range $|E|>2 D$, where in the absence of disorder there existed only mathematical (divergent) solutions having no physical interpretation. It is known that disorder extends the band, i.e. new physical states arise also at $|E|>2 D$. The question is: can delocalized states exist amongst these new states? The answer is simple. In the region $|E|>2 D$ only unbounded input signals $s_{n}^{(0)}$ exist. Such a signal cannot be transformed into a bounded output signal $s_{n}$. The output signal $s_{n}$ is always unbounded with a dual interpretation: on the one hand it corresponds to mathematical solutions (no physical interpretation); on the other hand, the divergence of the output signal can be associated with an emergence of the localized states. I.e., an emergence of delocalized states in the region $|E|>2 D$ (outside the band) is impossible [13].

Use of the filter function $H(z)$ is a general and abstract method for describing the metal-insulator transition, valid for any space dimension. Earlier [12] we used a less fundamental approach for the 1-D problem, based on the calculation of the Lyapunov exponent for the $\psi^{2}$-definition

$$
\gamma=\lim _{n \rightarrow \infty} \frac{1}{2 n} \ln s_{n}
$$

where for the 1-D case $s_{n} \equiv\left\langle\psi_{n}^{2}\right\rangle$. Taking into account that the divergence of the output signal $s_{n}$ is caused by the divergence of the filter $h_{n}$, one can use also the following equation

$$
\gamma=\lim _{n \rightarrow \infty} \frac{1}{2 n} \ln h_{n}
$$

In the 1-D case both definitions of $\gamma$ are equivalent, since for a fixed energy and disorder, for a second initial condition $\psi_{1}=\alpha$, one gets the single output signal $s_{n}$.

Let us use for illustration eqs.(39), (40). Restricting ourselves by the band center $E=0$ (which simplifies the equations) and making the inverse Ztransform, one gets 


$$
\begin{array}{r}
s_{n}=|\alpha|^{2} \frac{z_{0}}{\left(z_{0}^{2}+1\right)}\left[z_{0}^{n}-\left(-1 / z_{0}\right)^{n}\right], \\
h_{n}=\delta_{n, 0}+\frac{\left(z_{0}^{2}-1\right)}{\left(z_{0}^{2}+1\right)}\left[z_{0}^{n}-\left(-1 / z_{0}\right)^{n}\right], \\
z_{0}=\frac{\sigma^{2}+\sqrt{4+\sigma^{4}}}{2},
\end{array}
$$

provided $s_{n}^{(0)}=|\alpha|^{2}\left[1-(-1)^{n}\right] / 2$ (bounded input signal). Indeed, simultaneous divergence of the output signal $s_{n}$ and the filter $h_{n}$ arises due to the fact that the asymptotic behaviour of both quantities is determined by the same parameter, $z_{0}=\exp (2 \gamma)>1$.

However, this equivalence is no longer valid for a space dimension higher than one. Since the second initial condition is defined by the field $\alpha_{\mathbf{m}}$, this corresponds to a continuum of input signals $s_{n}^{(0)}$ and, respectively, a continuum of output signals $s_{n}$. That is, the definition (49) is not valid, since it is not clear that different signals should correspond to the same Lyapunov exponent $\gamma$. Formally, a whole continuum of solutions should be analyzed. However, eq.(35) demonstrates that the fundamental Lyapunov exponent $\gamma$ does not depend at all on the field $\alpha_{\mathbf{m}}$, it is sufficient to define the Lyapunov exponent using only eq.(50).

\subsection{Phase diagram and multiplicity of solutions}

A more detailed analysis $[12,13,16]$ reveals another problem of the definition (49): this is valid only in the 1-D case where for any disorder $\sigma$ only the phase of the localized solutions exists.

As it was mentioned above, for the calculation of the fundamental filter $h_{n}$ using the inverse Z-transform, eq.(38), the contour integration over the socalled region of convergence (ROC) [24] is necessary, provided the filter under consideration is causal. It is shown $[12,13]$ that dependent on the energy $E$ and disorder $\sigma$, two general cases are possible for the Anderson model with $D \geq 2$. In the first case, the ROC lies outside the circle $|z|>1$. The filter $h_{n}$ is thus unstable and describes the localized states (insulating phase). In the second case, the ROC consists of two domains in the complex plane: one domain is inside the circle $|z| \leq 1$, another one - outside this circle, $|z|>1$. Respectively, there are two ways to calculate the integral using eq. (38). The double solution arises, one describing delocalized states (filter $h_{n}^{(-)}$, metallic phase), the other localized states (filter $h_{n}^{(+)}$, insulating phase).

Consequently, in this range of parameters $E$ and $\sigma$ the two phases can co-exist, this is why the metal-insulator transition in the Anderson model has to be analyzed in terms of first-order phase transition theory $[12,13,16]$. It is known 
that complex (first or higher order) phase transitions introduced purely by noise can be generated for spatially extended systems [4]. For arbitrary random potentials the wave function can be either localized or delocalized (no co-existence!). However, in terms of statistics of an ensemble of random potentials, both localized and delocalized solutions can arise with a comparable probability. Namely this comparability of probabilities is the main characteristic of the first-order phase transition, in contrast to the second-order transitions where either a pure metallic phase (no localized states) or a pure insulating phase (no delocalized states) should exist: co-existence is impossible.

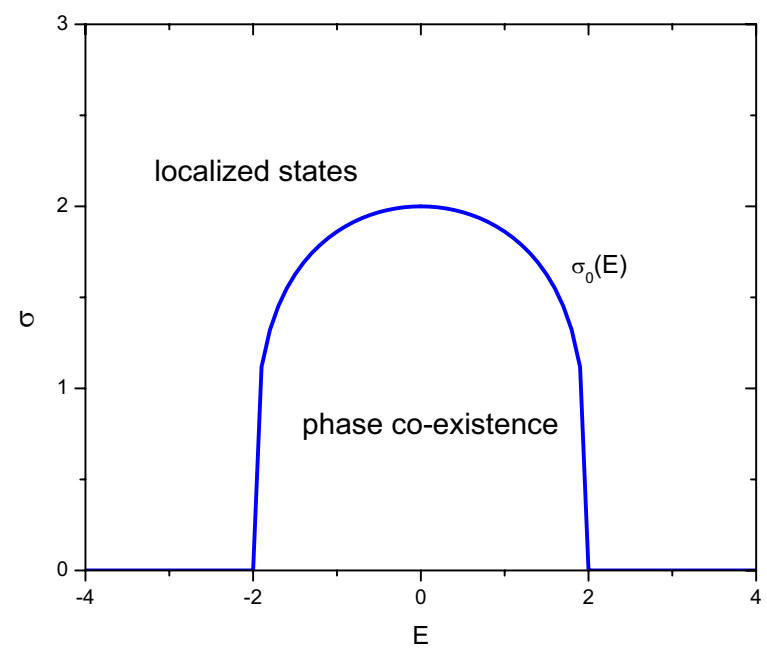

Fig. 2. Phase diagram for 2-D case.

For the illustration let us discuss here the phase diagram in terms of energy $E$ and disorder $\sigma$ for 2-D case as shown in Fig.2 (the band region $|E| \leq 4$ ). The other dimensions are discussed in Ref.[13]. The diagram in Fig.2 shows that all states with energies $|E|>2$ are localized at arbitrarily weak disorder. The localization at the finite disorder parameter $\sigma \geq \sigma_{0}(E)$, where according to Ref.[12]

$$
\sigma_{0}(E)=2\left(1-E^{2} / 4\right)^{1 / 4},
$$

is also observed in the energy range $|E| \leq 2$. In other words, only pure insulating phase (no delocalized states) takes place in these cases. The localized states are observed also in the rest of the phase diagram, namely, for small energies, $|E| \leq 2$, and small disorder, $\sigma<\sigma_{0}(E)$. However, as it was mentioned above, the localized states could be observed only for a certain realization of the random potentials. For other realizations the delocalized states occur in the same range of energy and disorder, $|E| \leq 2$ and $\sigma<\sigma_{0}(E)$.

Phase co-existence creates a serious problem of the choice of an adequate math- 
ematical formalism. Strictly speaking, when calculating the average quantities on the ensemble of different realizations of random potentials, the contributions from pure metallic and insulating phases are considered as equivalent. However, such heterophase averages have no physical sense $[12,13,16]$. In a two-phase system with first-order phase transition one is interested in properties of pure phases. From this point of view, the earlier introduced output signals $s_{n}$ are also heterophase averages. This is why the derivation of equations for signals is not a goal, but the tool for obtaining a more fundamental property - the filter $H(z)$ (or $\left.h_{n}\right)$. As was noted $[12,13,16]$, it is such a filter which reveals the multiplicity of solutions and which permits us to determine the phase diagram for the Anderson model.

\subsection{Averaging over initial conditions}

Let us discuss now the problem of averaging over initial conditions $[12,13]$. This procedure was introduced in Ref. [12], its meaning is quite simple. First of all, we choose a particular second initial condition, i.e. a field $\alpha_{\mathbf{m}}$. Secondly, let us consider the field $\alpha_{\mathbf{m}}^{\prime} \equiv \alpha_{\mathbf{m}+\mathbf{m}_{\mathbf{0}}}$ obtained from the first field as the result of a trivial translation in transversal direction by the vector $\mathbf{m}_{\mathbf{0}}$. It is obvious that the relevant diagonal correlators $\chi^{\prime}(n)_{\mathbf{m}} \equiv \chi(n)_{\mathbf{m}+\mathbf{m}_{\mathbf{0}}}$ can also be obtained by the argument shift in a transversal direction. Both solutions are physically equivalent. The Fourier transform of the field $\alpha_{\mathbf{m}}$ with the vector shift $\mathbf{m}_{\mathbf{0}}$ satisfies a simple relation: $\alpha^{\prime}(\mathbf{k}) \equiv \exp \left(-i \mathbf{k m}_{\mathbf{0}}\right) \alpha(\mathbf{k})$.

Eqs. (28) to (30) clearly demonstrate that the vector shift affects only signals for particular modes $\mathbf{k}$, and all physically equivalent solutions differ from each other only by phases:

$$
\begin{array}{r}
\chi^{\prime(0)}(z, \mathbf{k}) \equiv \exp \left(-i \mathbf{k m}_{\mathbf{0}}\right) \chi^{(0)}(z, \mathbf{k}) \\
\chi^{\prime}(z, \mathbf{k}) \equiv \exp \left(-i \mathbf{k m}_{\mathbf{0}}\right) \chi(z, \mathbf{k})
\end{array}
$$

Note that only the fundamental mode $\mathbf{k}=\mathbf{0}$ remains invariant. Averaging now the signal over all translations in transversal directions (averaging over initial conditions [12]) gives zero for all non-fundamental modes, $\mathbf{k} \neq \mathbf{0}: \overline{\chi^{(0)}(z, \mathbf{k})}=$ $0, \overline{\chi(z, \mathbf{k})}=0$. Nonzero is only the fundamental mode $(\mathbf{k} \equiv \mathbf{0}): \overline{\chi^{(0)}(z, \mathbf{0})}=$ $S^{(0)}(z), \overline{\chi(z, \mathbf{0})}=S(z)$. The average diagonal correlator loses its dependence on the argument $\mathbf{m}: \overline{\chi(n)_{\mathbf{m}}}=s_{n}$. That is, averaging over initial conditions is an efficient tool for getting rid of all non-fundamental modes which are non-essential for the analysis.

Note that eq. (36) contains $\Gamma(\mathbf{k})=|\alpha(\mathbf{k})|^{2}$ which is a function of the field $\alpha_{\mathbf{m}}$, its Fourier transform is $\Gamma_{\mathbf{m}}$. The averaging over initial conditions corresponds mathematically in the initial eq. (18) to the replacement of the initial condition 
$x(1)_{\mathbf{m}, \mathbf{l}}=\alpha_{\mathbf{m}} \alpha_{\mathbf{l}}$ by the average, $\overline{x(1)_{\mathbf{m}, \mathbf{l}}}=\Gamma_{\mathbf{m}-\mathbf{l}}$. After such a replacement of initial conditions the system becomes much simpler: it is translation-invariant in transversal directions which makes use of the double Fourier transform unnecessary. In other words, averaging over initial conditions is nothing but a simple mathematical trick which permits to get quickly the fundamental property of system - the filter $H(z)$. Such tricks are based on the fact that the filters are universal system characteristics, describing the solution transformation from order to disorder. Such universal characteristics are independent of the initial conditions and other details. The initial conditions determine such non-universal properties as signals. Consequently, in order to obtain the fundamental filter $H(z)$ and then the phase diagram, one can perform linear operations with signals, in particular, averaging over initial conditions.

Another example of such operations is the following. Let us consider the field $\alpha_{\mathbf{m}}$ not as fixed (second initial condition), but as a random variable, with simultaneous averaging over the field $\alpha_{\mathbf{m}}$ and over the ensemble of the random potential realizations $\varepsilon_{\mathcal{M}}$. We assume the absence of correlations between the potentials $\varepsilon_{\mathcal{M}}$ and the field $\alpha_{\mathbf{m}}$; unlike the correlation between the field components characterized by the arbitrary correlation function $\left\langle\alpha_{\mathbf{m}} \alpha_{\mathbf{l}}\right\rangle=\Gamma_{\mathbf{m}-\mathbf{l}}$. The initial condition in eq.(18) becomes $x(1)_{\mathbf{m}, \mathbf{l}}=\Gamma_{\mathbf{m}-\mathbf{l}}$. Taking into account that the operator $\mathcal{L}_{\mathbf{m}, \mathbf{m}^{\prime}}$ by its definition, eq.(3), depends only on the on the argument difference, $\mathbf{m}-\mathbf{m}^{\prime}$, one gets the translation invariant system, where $X(z)_{\mathbf{m}, \mathbf{l}} \equiv \hat{X}(z)_{\mathbf{m}-\mathbf{l}}$, provided for the diagonal correlators $X(z)_{\mathbf{m}, \mathbf{m}}=\chi(z)_{\mathbf{m}} \equiv$ $\hat{X}(z)_{\mathbf{0}}=S(z)$ holds (the diagonal correlators are $\mathbf{m}$-independent).

In this case in order to solve eq. (18), it is sufficient to use a single Fourier transform

$$
\hat{X}(z)_{\mathbf{m}}=\int \frac{d^{p} \mathbf{k}}{(2 \pi)^{p}} \hat{X}(z, \mathbf{k}) e^{-i \mathbf{k m}}
$$

Instead of eq.(22) one gets

$$
U(z, \mathbf{k},-\mathbf{k}) \hat{X}(z, \mathbf{k})=\Gamma(\mathbf{k})+\sigma^{2} S(z)
$$

which gives

$$
S(z)=\int \frac{d^{p} \mathbf{k}}{(2 \pi)^{p}} \hat{X}(z, \mathbf{k}) .
$$

As a result, one returns to the fundamental eqs.(34) to (37), with replacement of the $|\alpha(\mathbf{k})|^{2}$ for $\Gamma(\mathbf{k})$. 


\section{Conclusion}

We would like to stress that in this paper we presented a mathematically rigorous method for the calculation of the phase diagram for the Anderson localization in arbitrary dimensions, which was briefly discussed earlier $[12,13]$. The phase diagram for the metal-insulator transition is obtained using the Lyapunov exponent $\gamma$. Localized states correspond to values of $\gamma>0$, i.e. a divergence of the averages over wavefunctions. This divergence is mathematically similar to the divergence of averages for the diffusion motion. That is, transition to the localized states can be treated as a generalized diffusion. From this viewpoint, in order to determine the range of the existence of localized states (i.e. the phase diagram) and the type of localization (exponential or non-exponential), it is sufficient to solve equations for the joint correlators. We have shown that these equations are exactly solvable analytically.

In its turn, the appearance of the generalized diffusion arises due to the instability of a fundamental mode corresponding to correlators. The generalized diffusion can be described in terms of signal theory, which operates with the concepts of input and output signals and the filter function. Delocalized states correspond to bounded output signals, and localized states to unbounded output signals, respectively. Transition from bounded to unbounded signals is defined uniquely by the filter function $H(z)$, or more precisely, by the position

of its poles in the complex plane. This function can be calculated for arbitrary space dimension D.

\section{Acknowledgements}

V.N.K. gratefully acknowledges the support of the Deutsche Forschungsgemeinschaft. The authors are indebted to E. Kotomin for detailed discussions of the paper.

\section{References}

[1] G.W. Gardiner, Handbook of Statistical Methods for Physics, Chemistry and Natural Sciences (Springer, Berlin, 1997).

[2] N.G. Van Kampen, Stochastic Processes in Physics and Chemistry (Elsevier, North-Holland, Amsterdam, 1992).

[3] J. Qiang and S.Habib, Phys. Rev. E 62, 7430 (2000). 
[4] C. Van den Broeck, J. M. R. Parrondo, R. Toral, and R. Kawai, Phys. Rev. E 55, 4084 (1997).

[5] P.W. Anderson, Phys. Rev. 109, 1492 (1958).

[6] B. Kramer and A. MacKinnon, Rep. Prog. Phys. 56, 1469 (1993).

[7] M. Janssen, Phys. Rep. 295, 2 (1998).

[8] E. Abrahams, S.V. Kravchenko, M.P. Sarachik, Rev. Mod. Phys. 73, 251 (2001).

[9] I.M. Lifshitz, S.A. Gredeskul, and L.A. Pastur, Introduction to the Theory of Disordered Systems (Wiley, New York, 1988).

[10] M. Vojta, Rep. Prog. Phys., 66, 2069 (2003).

[11] R.J.Baxter, Exactly Solved Models in Statistical Mechanics (Academic Press, London, New York, 1982).

[12] V.N. Kuzovkov, W. von Niessen, V. Kashcheyevs and O. Hein, J. Phys.: Condens. Matter, 14, 13777 (2002).

[13] V.N. Kuzovkov and W. von Niessen, Eur. Phys. J. B, 42, 529 (2004).

[14] G. Radons, Physica D, 187, 3 (2004).

[15] P. Markoš, L.Schweitzer and M.Weyrauch, J. Phys.: Condens. Matter, 16, 1679 (2004).

[16] V.N. Kuzovkov, V Kashcheyevs, and W. von Niessen, J. Phys.: Condens. Matter, 16, 1683 (2004).

[17] L. Molinari. J.Phys.A: Math. Gen. 25, 513 (1992).

[18] J. B. Pendry, J. Phys. C: Solid State Phys., 15, 3493 (1982).

[19] I.M. Sokolov and J. Klafter, Chaos, 15, 026103 (2005).

[20] W. Ebeling and I.M. Sokolov, Statistical Thermodynamics and Stochastic Theory of Nonequilibrium Systems (Word Scientific, Singapore, 2005).

[21] E. A. Kotomin and V. N. Kuzovkov, Modern Aspects of Diffusion-Controlled Reactions: Cooperative Phenomena in Bimolecular Processes, Vol. 34 of Comprehensive Chemical Kinetics (Elsevier, North Holland, Amsterdam, 1996).

[22] S.B. Yuste and L. Acedo, Physica A, 336334 (2004).

[23] M.A. Muñoz and T. Hwa, Europhys. Lett., 41147 (1998).

[24] T.F. Weiss. Signals and systems. Lecture notes. http:// umech.mit.edu/ weiss/ lectures.html 